\title{
Epigenetics and Novel Therapeutic Approaches
}

\section{Nitai C. Hait*}

Department of Biochemistry and Molecular Biology, VCU School of Medicine, 1101 East Marshall Street, Richmond, Virginia 23298-0614, USA

Epigenetic changes are targeted to the DNA, as well as chromatin core histones and unlike genetic mutations these changes are reversible, making them attractive targets for therapeutic purposes [1]. Multiple levels of epigenetic regulatory mechanisms provide orchestration of chromatin structure for gene expression in healthy cells; however, result inappropriate silencing or activation of gene expressions, finally leading to various disease states such as cancer [2]. Epigenetic modifications can be a determinant factor to characterize the disease states, and detailed profiling of these modifications may allow predictions of disease outcome or therapeutic strategies. Epigenetic therapy can offer promising tools to restore/reverse changes. Interestingly, several epigenetic inhibitors such as DNA methyltransferase inhibitors (DNMTis), and histone deacetylase inhibitors (HDACis) approved by the US Food and Drug Administration (FDA) are in clinics for the treatment of various hematological malignancies. Potential epigenetic modifications, such as DNA methylation, covalent histone modifications, such as histone acetylation/deacetylation, histone methylation/demethylation, histone phosphorylation, non-covalent mechanisms, such as incorporation of histone variants, nucleosome remodeling and positioning, microRNAs (miRNAs) expression are important targets for therapeutic purposes $[2,3]$. How these effectors interact to each other to affect gene expression and cause disease is the active field of study in epigenetics.

\section{Epigenetic Mechanisms and Clinical Relevance}

DNA methylation is the most extensively studied epigenetic modification, provides a stable gene silencing and works in association with histone modifications. DNA (cytosine-5)-methyltransferase (DNMT)-3A and DNMT3B are responsible for de novo DNA methylation patterns, which are then copied to daughter cells during $S$ phase by DNMT1. DNMTs mostly act as oncoproteins. Cancer is characterized by global hypomethylation and hypermethylation at the $\mathrm{CpG}$ island contributes to tumorigenesis by silencing tumor suppressor genes [3]. Acetylation/deacetylation and methylation/demethylation of nucleosomal histones tails are critical components of an epigenetic indexing, that demarcating transcriptionally active or inactive chromatin domains for gene expression. An imbalance in the equilibrium of these modifications has been associated with carcinogenesis and cancer progression. Cancer cells are characterized by dysregulation of histone methyltransferases (HMTs) and histone demethylases (HDMs), overexpression of histone deacetylases (HDACs), and a global reduction of $\mathrm{H} 4 \mathrm{~K} 16 \mathrm{ac}$ and $\mathrm{H} 4 \mathrm{~K} 20 \mathrm{me}$. HDACis have long been studied in the clinical setting as potential therapies and development of HDACis has become the subject of intense interest. Several HDACis are FDA approved, including the hydroxamic acid-based compound SAHA and the depsipeptide romidepsin, whereas others are currently in clinical trials for cancer (phenylbutyrate and entinostat) and neurologic diseases (entinostat) [3]. Alterations in H3K9 and H3K27 methylation patterns are associated with aberrant gene silencing in various forms of cancer. EZH2, H3K27 HMT, inhibition had been shown as a potential therapeutic strategy for lymphoma [4]. Increased levels of G9a, another H3K9 HMT, have been found in liver cancer and are implicated in perpetuating malignant phenotype possibly through modulation of chromatin structure. MLL, the H3K4 HMT, lead to ectopic expression of various homeotic (Hox) genes and play a key role in leukemic progression. LSD1, the first identified lysine demethylase, increased levels of $\mathrm{H} 3 \mathrm{~K} 4 \mathrm{me} 2$ and $\mathrm{H} 3 \mathrm{~K} 9 \mathrm{ac}$ histone modifications, reduced $\mathrm{H} 3 \mathrm{~K} 9 \mathrm{me} 2$ modification and promoted expression of $\mathrm{p} 53$ target genes (p21 and PUMA), leading to apoptosis of glioma xenograft tumors [3]. The never-ending lists of specific HMTs and HDMs are deregulated in cancers and have therapeutic potential. In addition, targeting histone $\mathrm{H} 3$ phosphorylation by histone-modifying enzymes may have therapeutic potential for cancer treatment. Nucleosome positioning and histone variants (H2A.Z and H3.3) incorporation into the nucleosomes is greatly enhanced the complexity of the landscape of epigenetic gene silencing in cancer. $\mathrm{H} 3.3$ and H2A.Z are preferentially enriched at promoters of active genes. H2A.Z is overexpressed in several types of cancer and has been associated with the promotion of cell cycle progression. Therefore, histone variants as potential drivers of cancer initiation and/or progression, and, therefore, targeting histone deposition or the chromatin remodeling machinery may be of therapeutic value [5]. MicroRNAs (miRNAs) are able to induce heritable changes in gene expression without altering DNA sequence and thus contribute to the epigenetic landscape. In addition, miRNAs can both regulate and be regulated by other epigenetic mechanisms. Expression of miRNAs is dysregulated in several diseases including cancer and certain neurodegenerative disorders [3]. For example, miR-101 degrades EZH2 is down regulated in several types of cancer, leading to increased EZH2 expression (and consequently higher H3K27me3 levels) and decreased expression of tumor suppressor genes. Interestingly, miR-506 can suppress epithelial-mesenchymal transition (EMT), and reduce tumor growth in serous ovarian cancer mouse model [6].

\section{Concluding Remarks}

Epigenetics is the most rapidly expanding field in biology. Detailed understanding of epigenetic processes and mapping the epigenetic marks in disease states compare to the normal mammalian cells will allow designing more effective treatment strategies. Further, development of very specific epigenetic drugs with fewer side effects will be used with great hope to reset the abnormal epigenome in disease states such as cancer.

\section{References}

1. Reik W, Dean W, Walter J (2001) Epigenetic reprogramming in mammalian development. Science 293: 1089-1093.

2. Egger G, Liang G, Aparicio A, Jones PA (2004) Epigenetics in human disease

${ }^{*}$ Corresponding author: Nitai C. Hait, Department of Biochemistry and Molecula Biology, VCU School of Medicine, 1101 East Marshall Street, Richmond, Virginia 23298-0614, USA, Tel: 804-828-9333; E-mail: nchait@vcu.edu

Received July 26, 2013; Accepted July 26, 2013; Published July 30, 2013

Citation: Hait NC (2013) Epigenetics and Novel Therapeutic Approaches. J Mo Genet Med 7: 74. doi:10.4172/1747-0862.1000074

Copyright: (c) 2013 Hait NC. This is an open-access article distributed under the terms of the Creative Commons Attribution License, which permits unrestricted use, distribution, and reproduction in any medium, provided the original author and source are credited 
and prospects for epigenetic therapy. Nature 429: 457-463.

3. Kelly TK, De Carvalho DD, Jones PA (2010) Epigenetic modifications as therapeutic targets. Nat Biotechnol 28: 1069-1078.

4. McCabe MT, Ott HM, Ganji G, Korenchuk S, Thompson C, et al. (2012) EZH2 inhibition as a therapeutic strategy for lymphoma with EZH2-activating mutations. Nature 492: 108-112.
5. Vardabasso C, Hasson D, Ratna kumar K, Chung CY, Duarte LF, et al. (2013) Histone variants: emerging players in cancer biology. Cell Mol Life Sci.

6. Yang D, Sun Y, Hu L, Zheng H, Ji P, et al. (2013) Integrated analyses identify a master microRNA regulatory network for the mesenchymal subtype in serous ovarian cancer. Cancer Cell 23: 186-199. 